Behzad Mesbahzadeh, Seyed Ali Rajaei, Parnia Tarahomi, Seyed Ali Seyedinia, Mehrnoush Rahmani, Fatemeh Rezamohamadi, Muhammad Azam Kakar, Nasroallah Moradi-Kor*

\title{
Beneficial effects of Spirogyra Neglecta Extract on antioxidant and anti-inflammatory factors in streptozotocin-induced diabetic rats
}

https://doi.org/10.1515/bmc-2018-0015

received October 29, 2018; accepted November 19, 2018.

Abstract: Objectives: This study was conducted to evaluate the effects of oral supplementation of Spirogyra algae on oxidative damages and inflammatory responses in streptozotocin (STZ)-induced diabetic rats. Methods: Diabetes was induced by administration of $55 \mathrm{mg} / \mathrm{kg}$ of streptozotocin. A total of sixty-four rats were divided into eight groups of eight rats each as follows:1) nondiabetic control; 2, 3, and 4) non-diabetic rats treated with 15, 30, and $45 \mathrm{mg}$ of Spirogyra algae $/ \mathrm{kg} / \mathrm{d}$; 5) control diabetic; and 6, 7, and 8) diabetic rats treated with 15, 30, and $45 \mathrm{mg}$ of Spirogyra algae extract. At the end of the trial, the serum concentrations of glucose, interleukin-6 (IL-6), tumor necrosis factor-a (TNF-a), malondialdehyde (MDA), glutathione (GSH), total antioxidant status (TAS), C-reactive protein (CRP), insulin, triglycerides, and cholesterol were examined by specified procedures. Results: Our findings indicated that the administration of STZ significantly increased the serum concentrations of glucose, triglycerides, cholesterol, CRP, IL-6, TNF-a, and MDA and decreased the serum levels of GSH and TAS $(\mathrm{P}<0.05)$ in diabetic rats. Oral administration of Spirogyra alleviated adverse effects of diabetes on

\footnotetext{
*Corresponding author: Nasroallah Moradi-Kor, Student Research Committee, Faculty of Medicine, Semnan University of Medical Sciences, Semnan, Iran; Research Centre of Physiology, Faculty of Medicine, Semnan University of Medical Sciences, Semnan, Iran, E-mail: moradikor.nasroallah@yahoo.com Behzad Mesbahzadeh: Cardiovascular Diseases Research Center, Birjand University of Medical Sciences, Birjand, Iran Seyed Ali Rajaei: Oral and Maxillofacial Surgeon, Faculty of Dentistry, Semnan University of Medical Sciences, Semnan, Iran Parnia Tarahomi, Seyed Ali Seyedinia, Mehrnoush Rahmani, Fatemeh Rezamohamadi: Student Research Committee, Faculty of Medicine, Semnan University of Medical Sciences, Semnan, Iran Muhammad Azam Kakar: Director Planning and Development, L\&DD Department, Spinny Road Quetta, Balochistan, Pakistan
}

oxidative stress and inflammatory factors in diabetic rats $(\mathrm{P}<0.05)$. Conclusion: It can be stated that Spirogyra algae extract can be used for treatment of diabetes likely due to prevention of oxidative stress and alleviation of inflammation in the rat model.

Keywords: Antioxidant status; Diabetes; Glutathione; Inflammatory parameters; Malondialdehyde; Rat.

\section{Introduction}

Diabetes mellitus (DM) is a metabolic disorder and it is classified into two types, type 1 and type 2. Type 2 diabetes mellitus is broadly related with hyperglycemia and insulin resistance ${ }^{1}$. Insulin resistance in some tissues in the body, such as skeletal muscle, adipose tissue, and the liver, causes the development of diabetes ${ }^{2}$. It is reported that a positive correlation exists between levels of proinflammatory cytokines tumor necrosis factor-a (TNF-a), interleukin-1 (IL-1), and IL-6 with insulin ${ }^{3}$. An interesting study has suggested that inflammation can directly causes pathogenesis of insulin resistance ${ }^{4}$. TNF- $\alpha$ causes inflammation and decreases $\beta$-cell secretion of insulin ${ }^{5}$. Additionally, TNF- $\alpha$ increases triglycerides production ${ }^{6}$. On the other hand, oxidative stress is created due to an imbalance between oxidant and antioxidant systems, increase of free radical production, and reduction in activity of antioxidants ${ }^{7}$. Oxidative stress induces hyperglycemia, promotes diabetes, and causes other severe complications ${ }^{8}$. Antioxidants can scavenge free radicals and reactive oxygen species (ROS) through inhibiting lipid peroxidation and decreasing the adverse effects produced by ROS ${ }^{9,10}$. Generally, synthetic drugs are used to treat the diabetes, but their use is faced with major limitations due to their many side effects. Natural compounds have recently been tried in many regions with mixed results. In this context, algae are major source of natural compounds 
that are broadly found in nature. Algae are known to have antibacterial, antiviral, antioxidant, anti-inflammatory properties, and cytotoxic activities ${ }^{11}$. Polysaccharides in algae are also found to scavenge free radicals under in vivo and in vitro conditions ${ }^{12}$. Spirogyra spp. of algae are filamentous freshwater green algae that contain high amounts of protein, carbohydrate, fat, sulfate, and dietary fiber ${ }^{13}$. Moreover, the Spirogyra produce compounds such as alkaloids, steroids, flavonoids, tannins and terpanoids ${ }^{14}$ Spirogyra neglecta (SN) is reported to have antioxidant activity ${ }^{15}$ and anti-inflammatory effects ${ }^{16}$. Keeping in view the above mentioned studies, it was hypothesized that $\mathrm{SN}$ algae can be used to treat the diabetes patients due its known antioxidant and anti-inflammatory properties. Thus, this study was conducted to evaluate the effects of SN algae on oxidative stress and inflammation in diabetic the rat model.

\section{Materials and Methods}

\section{Animals}

Adult male Wistar rats, weighing $200 \pm 20 \mathrm{~g}$ and 9 wks of age, were purchased from Pastur Institute, Tehran-Iran. Sixtyfour Wistar rats were randomly assigned into 8 groups $(\mathrm{n}=8)$. Animals were further divided into four groups for diabetic and four groups for non-diabetic. Animal in the diabetic groups received 0 (control), 15,30 , or $45 \mathrm{mg} / \mathrm{kg}$ of SN extract. Algae extract was administrated in gavage form for 42 days after induction of diabetes. Animals in healthy, nondiabetic groups orally received 0 (control), 15 , 30 or $45 \mathrm{mg} / \mathrm{kg}$ of SN extract. Rats were grouped in a wellventilated animal facility in stainless steel cages in order to allow them free mobility. Rats received a standard feed (Javaneh Khorasan, Iran) and fresh water. Rats were kept under controlled conditions including temperature $(22 \pm$ $\left.2^{\circ} \mathrm{C}\right)$ and humidity $(55 \pm 5 \%)$. A lighting diet $(12 \mathrm{~h} \mathrm{light} / 12 \mathrm{~h}$ dark) was considered. All the experiments were conducted in accordance with the National Institutes of Health Guide (NIH Publication No. 85-23, revised 1996) for the Care and Use of Laboratory Animals.

\section{Preparation of Spirogyra neglecta extract}

Spirogyra neglecta was provided from Bistoon pond (Kermanshah-Iran) and dried for $10 \mathrm{~d}$. The extract was prepared as described by Ontawong et al. ${ }^{15}$. Lyophilized extract were maintained in $4^{\circ} \mathrm{C}$ prior to experiments.

\section{Pilot study}

Forty Wistar rats were fasted for $24 \mathrm{~h}$ and then divided into 8 groups with 5 animals each. Animals were orally administrated with $0.2 \mathrm{~mL}$ distilled water and a dose of SN extract at either 10, 15, 30, 45, 60, 70, or $90 \mathrm{mg} / \mathrm{kg}$. The rats were observed in order to assess the behavioral responses, toxicity signs, and mortality for $48 \mathrm{~h}$. We did not observe any mortality but diarrhea was observed in the 70 and $90 \mathrm{mg} / \mathrm{kg}$ test groups. Thus, we selected doses of 15, 30, and $45 \mathrm{mg} / \mathrm{kg}$ for further studies. These doses were not observed to have any behavioral responses and signs of toxicity.

\section{Induction of Diabetes}

In order to induce diabetes, animals were intraperitoneally administrated with $55 \mathrm{mg} / \mathrm{kg}$ of streptozotocin (STZ, Sigma, St. Louis, MA, USA) in $0.1 \mathrm{M}$ citrate buffer $\mathrm{pH} 4.5$ as described by Jaiswal et al. ${ }^{17}$. Rats were bled through the tail in order to confirm induction of diabetes, and assessed by a glucometer (Accu-Check, Roche, Manheim, Germany). Rats with a glucose level $>250 \mathrm{mg} / \mathrm{dl}$ were considered diabetic ${ }^{17}$.

\section{Blood sampling and antioxidant, inflammatory and blood variables}

On day 42 after interventions and $24 \mathrm{~h}$ fasting, animals were intraperitoneally anaesthetized by administration of sodium pentobarbital injection $(60 \mathrm{mg} / \mathrm{kg})$. Blood samples were collected into tubes without heparin. The obtained samples were centrifuged at $4000 \mathrm{~g}$ for $10 \mathrm{~min}$ in $4^{\circ} \mathrm{C}$ and sera were stored in $-20^{\circ} \mathrm{C}$ for subsequent trials. An enzyme-linked immunosorbent assay (ELISA) was used to evaluate the levels of TNF-a and IL- 6 as recommended by the manufacturer's instructions. In short, rat TNF $\alpha$ and IL-6 specific-specific monoclonal antibodies were loaded into 96-well plates. The rat specific detection polyclonal antibodies were biotinylated. The samples test and biotinylated detection antibodies were added to the wells subsequently and then continued through washing with PBS or TBS buffer. Avidin-Biotin-Peroxidase Complex was included and unbound conjugates were investigated away with PBS or TBS buffer. TMB catalyzed by HRP used to produce a blue color product which altered into yellow in following inclusion of acidic stop solution. The density of yellow was proportional rather than TNFa level of sample captured in plate. TBARS (thiobarbituric acid 
reactive substances) procedure was used to evaluate the serum malondialdehyde (MDA) as described by others ${ }^{18}$. The levels of glutathione (GSH) was evaluated by Ellman's reagent (5, 50-dithio-bis-2-nitrobenzoic acid,) as reported by previous studies ${ }^{19}$. The total antioxidant status (TAS) was assessed by FRAP (ferric reducing antioxidant power) procedure ${ }^{20}$. C-reactive protein (CRP) was measured by specific kits (The Invitrogen CRP ELISA Kit, Camarillo, CA). The serum concentrations of glucose, cholesterol, triglycerides, and insulin were assessed by commercial kits (Pars Azmoon-Iran).

\section{Statistical analysis}

The data were analyzed by using GraphPad Prism statistical software (GraphPad Software, Inc., La Jolla, CA, USA) by one-way/two way analysis of variance (ANOVA) followed by Dunnett's Multiple Range Test. Animals in healthy group and diabetic group were separately compared by T-test. The findings are shown as mean \pm SD. A value of $P<0.05$ was considered to be statistically significant.

\section{Results}

\section{Antioxidant variables}

Effects of different levels of SN extract on MDA, GSH, and TAS are shown in Figure 1. Results showed that induction of diabetes significantly increased levels of MDA (Figure 1A), and decreased levels of GSH and TAS $(P<0.0001)$ (Figure 1B and C, control diabetic vs. control non-diabetic). The different doses of $\mathrm{SN}$ had no significant effects on MDA, GSH, and TAS in non-diabetic groups ( $P$ $>0.05)$. In diabetic rats, with increasing doses of extract, serum levels of MDA decreased and levels of GSH and TAS increased $(P<0.0001)$. The best responses were observed in 15,30 , and $45 \mathrm{mg} / \mathrm{kg}$ of $\mathrm{SN}$ extract in diabetic group, respectively.

\section{Inflammatory variables}

The serum concentrations of TNF-a, IL-6, and CRP were significantly increased in control diabetic in comparison to non-diabetic groups (Figure $2, P<0.0001$ ). Oral administration of SN extract especially in higher doses reduced the levels of TNF-a, IL-6, and CRP.
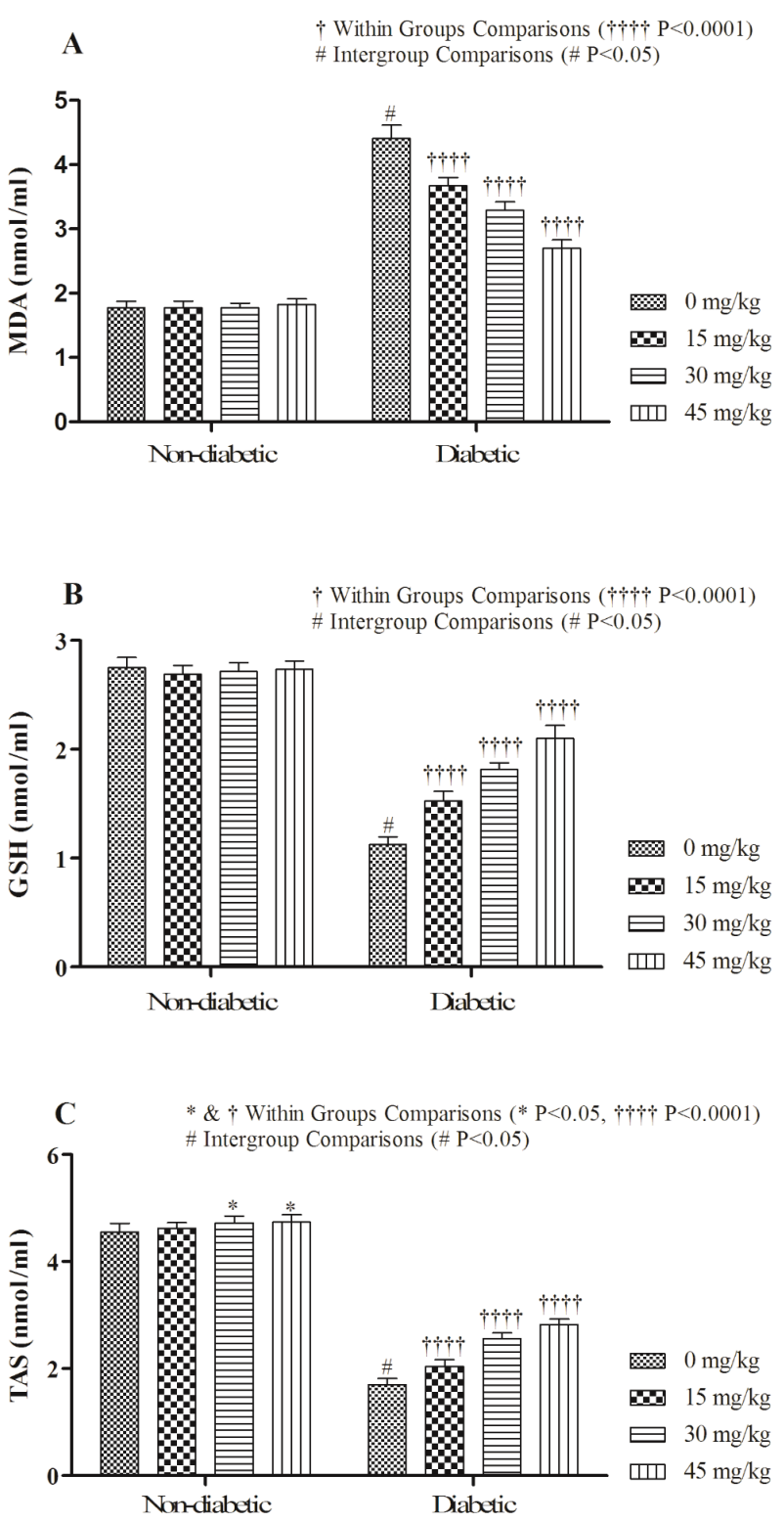

Figure 1: Effects of different levels of SN extract on antioxidant variables in diabetic and non-diabetic rats

\section{Blood variables}

The serum concentrations of cholesterol, triglycerides, and glucose were significantly increased and insulin concentration was decreased in diabetic rats in comparison to non-diabetic rats (Figure 3, $P<0.0001$ ). Diabetic rats treated with SN extract showed lower levels for cholesterol, triglycerides, and glucose and higher levels for insulin in comparison to diabetic control $(P<$ 0.0001). Better responses were observed in diabetic rats treated with higher doses (30 and $45 \mathrm{mg} / \mathrm{kg}$ ). 

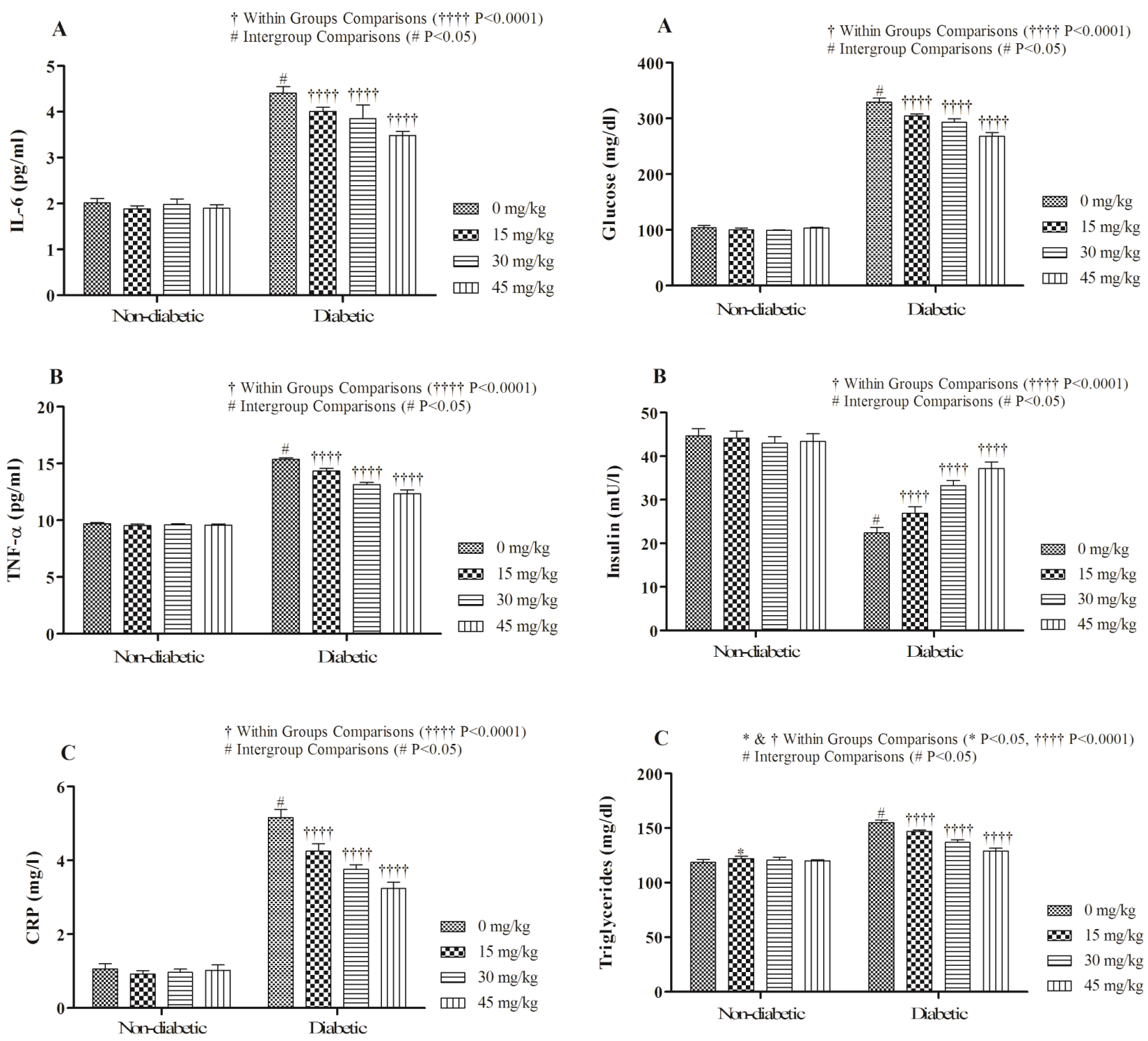

Figure 2: Effects of different levels of SN extract on inflammatory variables in diabetic and non-diabetic rats

\section{Discussion}

In this study, we have evaluated the pro-oxidantantioxidant balance by assessing MDA, GSH, and TAS levels in the diabetic and non-diabetic rats. Comparison of control diabetic and healthy rats showed a balance change toward pro-oxidation in STZ-induced diabetic animals. This resultantly increased the MDA, and decreased the GSH and TAS in rats. Previous published studies have shown that STZ induces diabetes and increases susceptibility to lipid peroxidation ${ }^{7,21}$. This might explain the phenomena behind the oxidative stresses induced diabetes and pathogenesis of diabetes. In this relation,

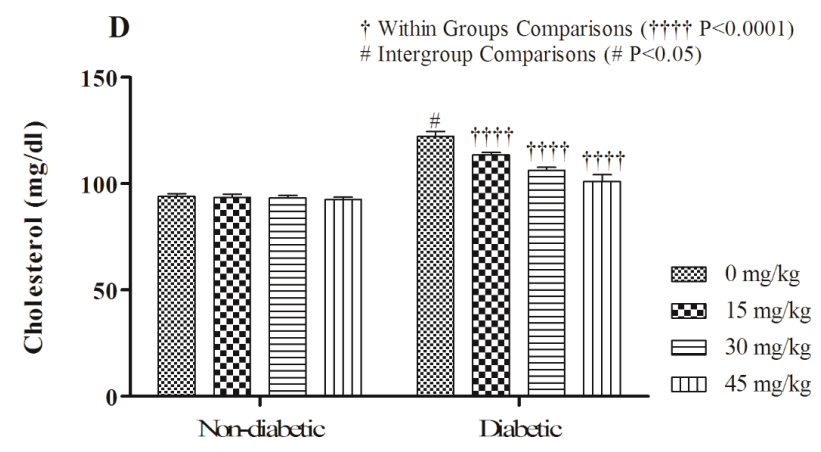

Figure 3.Effects of different levels of SN extract on blood variables in diabetic and non-diabetic rats.

the MDA is a product of lipid peroxidation that is rapidly combined with biomolecules and deregulates glucose metabolism ${ }^{22}$. In the present study, oral administration of SN extract in diabetic rats improved antioxidant status 
due to increased GSH and TAS, and decreased MDA. This could be attributed to antioxidant components found in SN extract. SN extract is well known to have antioxidant properties due to its polyphenols contents ${ }^{23,24}$. In a relative study, Ontawong et $a .^{15}$ showed that SN extract can directly scavenge free radicals and, thus, decrease MDA. It is well known in the literature that improved antioxidant status decreases lipid peroxidation and MDA levels. It can be speculated that SN extract is increasing the TAS, and ultimately the GSH could decrease levels of MDA.

On othe hand, the serum concentrations of TNF-a, IL-6, and CRP were significantly increased in diabetic rats, but oral administration of SN extract especially in higher doses reduced the levels of TNF-a, IL-6, and CRP. Oxidative stress causes production of abnormal cytokine production (TNF-a and IL-6) ${ }^{25}$. TNF-a not only increases adipocyte lipolysis but also has adverse effects on the insulin signaling pathway through changing the tyrosine/ serine phosphorylation of insulin receptor substrate ${ }^{26}$. CRP is a key inflammatory factor that is regulated by IL-6, IL-1, and TNF- $\alpha$, and produced by the liver in response to inflammation ${ }^{27}$. In relation to this, Virgolici et al. ${ }^{28}$ showed that increased levels of inflammation were related with the increased oxidative stress. We also observed and noticed that diabetes induces oxidative stress in rats. In addition, Chang et al. ${ }^{29}$ have reported that diabetes mellitus may induce nuclear factor kappa B (NF-kB) that is responsible for the production of some proinflammatory cytokines. In our study, the oral treatment of SN extract interestingly decreased the levels of inflammatory cytokines. Similarly, Nasirian et al. ${ }^{30}$ reported that the oral gavage of Spirulina platensis microalgae reduced inflammatory factors in diabetic rats. Improved inflammatory parameters in response to SN extract can be attributed to antioxidant activity of polyphenolic compounds existing in SN extract that prevents the development of ROS. As mentioned earlier, SN extract has antioxidant activity that may prevent production of inflammatory cytokines. Nevertheless, greater antioxidant activity was observed in higher levels of its doses in the rat model.

Hypertriglyceridemia and hypercholesterolemia are common signs in diabetes ${ }^{31}$. Previous studies have also shown that STZ increases the sensitivity to lipid peroxidation ${ }^{7,21}$. In addition, this might be the phenomena in which the STZ enters into pancreatic $\beta$ cell through the low-affinity glucose protein-2 transporter. The STZ damages insulin-producing islet $\beta$-cells and decreases insulin secretion ${ }^{7}$. In this complex pathway system, the reduced hepatic insulin sensitivity may be caused by an increase in hepatic gluconeogenesis, postprandial hyperinsulinemia, and increased production of triglycerides in the liver cells.
SN extract has alleviated hyperglycemia, dyslipidemia and increased the insulin sensitivity in the rat model with diabetes mellitus ${ }^{33}$. Thus, TNF- $\alpha$ over produces triglycerides in plasma ${ }^{6}$. TNF- $\alpha$ reduces glucose intake of peripheral tissues through targeting insulin signaling pathways and glucose transporter 4 (GLUT4) ${ }^{32}$. In the present study, oral supplementing of SN extract have decreased the levels of TNF- $\alpha$. This might improve the levels of insulin and glucose through decreasing TNF- $\alpha$. Moreover, the oxidative stress and reactive oxygen species also degrades the lipids and increases the lipid peroxidation and ultimately leads to lipolysis. It can be concluded that SN extract decreased the levels of lipids probably by antioxidant properties in the rat model.

\section{Conclusion}

Our overall findings showed that STZ increased inflammatory and lipid variables in diabetic rat model. While decreasing antioxidant variables and levels of insulin. Oral treatment of SN extract has not cause any significant changes in the inflammatory, antioxidant, insulin, or lipid variables in non-diabetic rats. Oral gavage of SN extracts could decrease levels of MDA, lipid peroxidation and inflammatory variables in diabetic rats. It could be concluded that the $\mathrm{SN}$ extracts improved blood biochemical variables probably by acting on antioxidant pathway system and thus reducing inflammatory variables. Since levels of $45 \mathrm{mg} / \mathrm{kg}$ of SN extract in the rat model have better effects, we therefore recommend the same or slightly higher levels for treatment and management of diabetes in future trials.

Author contributions: All authors contributed toward data analysis, drafting and revising the paper and agree to be accountable for all aspects of the work.

Disclosure: The authors report no conflicts of interest in this work.

\section{References}

1. American Diabetes Association, "Diagnosis and classification of diabetes mellitus," Diabetes Care, vol. 27, suppl1, pp. s5s10, 2012.

2. Virally M, Blicklé JF, Girard J, Halimi S, Simon D, Guillausseau PJ. Type 2 diabetes mellitus: epidemiology, pathophysiology, unmet needs and therapeutical perspectives. Diabetes Metab. 2007;33: 231-44. 
3. Uysal KT, Wiesbrock SM, Marino MW, Hotamisligil GS. Protection from obesity-induced insulin resistance in mice lacking TNF-alpha function. Nature. 19;389:610-614.

4. Sun X, Han F, Yi J, Lina H, Ben W. Effect of Aspirin on the Expression of Hepatocyte NF-KB and Serum TNF- $\alpha$ in Streptozotocin-Induced Type 2 Diabetic Rats. Korean Med Sci. 2011;26:765-770.

5. Pankow JS, Duncan BB, Schmidt MI, Ballantyne CM, Couper DJ, Hoogeveen RC, Golden SH. Atherosclerosis Risk in Communities Study. Fasting plasma free fatty acids and risk of type 2 diabetes: the atherosclerosis risk in communities study. Diabetes Care. 2004;27:77-82.

6. Qin B, Anderson RA, Adeli K. Tumor necrosis factor- $\alpha$ directly stimulates the overproduction of hepatic apolipoprotein B100containing VLDL via impairment of hepatic insulin signaling. Am J Physiol Gastrointest Liver Physiol. 2008;294(5):1120-1129.

7. Samarghandian S, Farkhondeh T, Samini F, Borji A. Protective effects of carvacrol against oxidative stress induced by chronic stress in rat's brain, liver, and kidney. Biochem Res Int. 2016;2016:2645237.

8. Matough FA, Budin SB, Hamid ZA, Alwahaibi N, Mohamed J. The role of oxidative stress and antioxidants in diabetic complications. Sult Qaboos Univ Med. J. 2012;12:5-18.

9. Nasri H, Baradaran A, Shirzad H, Rafieian-Kopaei M. New concepts in nutraceuticals as alternative for pharmaceuticals. Int J Prev Med. 2014;5,1487-1499.

10. Al-malki AL, El-Rabey HA. The antidiabetic effect of low doses of Moringaoleifera Lam. seeds on streptozotocin induced diabetes and diabetic nephropathy in male rats. Bio Med Res Int. 2015; Article ID 381040, 13 pages.

11. Lordan S, Ross RP, Stanton C. Marine bioactives as functional food ingredients: potential to reduce the incidence of chronic diseases. Mar Drugs. 2011;9: 1056-1100.

12. Jiao G, Yu G, Zhang J, Ewart SE. Review of chemical structures and bioactivities of sulfated polysaccharides from marine algae. Mar Drugs. 2011;9:196-223.

13. Phinyo K, Khanongnuch C, Pekkoh J, Pumas C, Peerapornpisal Y. Nutritional values and polysaccharides in Tao (Spirogyra neglecta (Hassall) Kützing) from Phare Province Proceeding, The $2^{\text {nd }} M$ JU-Phrae National Research Conference. 2012;50-56.

14. Ratikanga A, Gitu LM and Oyaro N. Mineral composition, antioxidants and antimicrobial actibities of freshwater algae (spirogyra genus) from Jomo Kenyatta University of Agriculture and Technology (JKUAT). World Rural Observ. 2014;6(2):86-91.

15. Ontawong A, Saowakon N, Vivithanaporn P, Pongchaidecha A, Lailerd N, Amornlerdpison D, Lungkaphin A, and Srimaroeng C. Antioxidant and renoprotective effects of Spirogyra neglecta (Hassall) Kützing extract in experimental Type 2 diabetic rats. Bio Med Res Int. vol. 2013, Article ID 820786, 15 pages.

16. Amornlerdpison K, Duangjun D, Kanjanapothi T, Taesotikul Y. Peerapornpisal, Gastroprotective activity of Spirogyra neglecta (Hassall) K"utzing. KKU Scie J. 2012;40(1):236-241.

17. Jaiswal D, Rai PK, Mehta S, Chatterji S, Shukla S, Rai DK, Sharma G, Sharma B, Watal G. Role of Moringaoleifera in regulation of diabetes-induced oxidative stress. Asian Pac J Trop Med. 2013;6:426-432.

18. Biswas D1, Banerjee M, Sen G, Das JK, Banerjee A, Sau TJ, Pandit S, Giri AK, Biswas T. Mechanism of erythrocyte death in human population exposed to arsenic through drinking water. Toxicol Appl Pharmacol. 2008;230:57-66.
19. Beutler E, Duron O, Kelly BM. Improved method for the determination of blood glutathione. J Lab Clin Med. 1963;61:882-888.

20. Budin SB, Othman F, Louis SR, Bakar MA, Das S, Mohamed J. The effects of palm oil to cotrienol rich fraction supplementation on biochemical variables, oxidative stress and the vascular wall of streptozotocin-induced diabetic rats. Clinics (Sao Paulo). 2009;64:235-244.

21. Yazdanparast R, Ardestani A, Jamshidi S. Experimental diabetes treated with Achilleasantolina: effect on pancreatic oxidative parameters. J Ethnopharmacol. 2007;112:13-18.

22. Sivaraman K, SenthilKumar GP, Sankar P, Bobby Z. Attenuation of oxidative stress, inflammation and insulin resistance by Allium sativum in fructose-fed male rats. J Clin Diagn Res. 2013;7:1860-1862.

23. Rattanapot T, Mengumphan K, Srimaroeng C, Junthip R, Amornlerdpison D. Antioxidant activity of Spirogyra sp. and effect of its supplementation on growth performance of Tilapia in cage culture. J Fish Tech Res. 2012;6(2):23-34.

24. Junthip R, Amormledpison D, Chimsook T. Phytochemical screening, antioxidant activity and total phenolic content of Spirogyra spp. Adv Mater Res. 2013;699:693-697.

25. Shahady E. Hyperlipidemia in diabetes etiology, consequences and treatment. Circulation. 1995;91:2844-2850.

26. El-Abhar HS, Schaalan MF. Topiramate-induced modulation of hepatic molecular mechanisms: an aspect for its anti-insulin resistant effect. PLoS One. 2012;7(5):e37757.

27. Nicklas BJ, You T, Pahor M. Behavioral treatments for chronic systemic inflammation: effects of dietary weight loss and exercise training. CMAJ. 2005;172:1199-1209.

28. Virgolici B, Mohora M, Gaman L, Lixandru D, Manolescu B, ComanA, Stoian I. Relation between inflammation and oxidative stress markers in diabetic foot patients. Romanian. J Biophys. 2008;18(4):273-282.

29. Chang CC, Chang CY, Huang JP, Hung LM. Effect of resveratrol on oxidative and inflammatory stress in liver and spleen of streptozotocin-induced type 1 diabetic rats. Chinese J Physio. 2012;55(3):192-201.

30. Nasirian F, Dadkhah M, Moradi-kor N, Obeidavi Z. Effects of Spirulina platensis microalgae on antioxidant and antiinflammatory factors in diabetic rats. Diabetes, Metabolic Syndrome and Obesity: Targets and Therapy. 2018;11:375-380.

31. Khan BA, Abraham A, Leelamma S. Hypoglycemic action of Murraya koenigii (curry leaf) and Brassica juncea (mustard): mechanism of action. Indian J Biochem Biophys.1995;32(2):106-108.

32. Mostafa AM, Mohamed WS, Serwah AHA, Serwah MA. Effect of Diclofenac on Plasma Glucose level, Insulin Resistance, Inflammatory Markers and Hepatocytes in Diabetic Albino Rats. The Egyptian J Hospital Med. 2014;54:117-128.

33. Jitprawet N, Keapai W, Apichai S. Evaluation of antihyperglycemic and antihyperlipidemic potential of Spirogyra neglecta extract on type 2 diabetic rats," in Proceedings of the 1st ASEAN Plus Three Graduate Research Congress, p. HS 415, The Graduate School, ChiangMai University, ChiangMai, Thailand, 2012. 